\title{
Markers of somatic embryogenesis of Pinaceae species in in vitro culture (cytohistological, physiological and molecular genetic)
}

Tretyakova I.N.*, Park M.E.

V.N. Sukachev Institute of Forest SB RAS, Federal Research Center "Krasnoyarsk Science Center SB

RAS" Siberian Branch of the Russian Academy of Science, Krasnoyarsk, Russia

*email: culture@ksc.krasn.ru

Somatic embryogenesis in in vitro culture is an ideal model for studying cytohistological, physiological, and molecular genetic events that occur during the embryogenesis of a tree organism. The first peculiarity of embryogenic culture (EC) in Pinaceae species is the elongation, polarization, and asymmetric division of somatic cells, as well as the localization of IAA at one end of the elongated cell. Further, a well-developed embryogenic tissue is formed, represented by the embryonal-suspensor mass (ESM), in which there is an active formation of globular somatic embryos through cleavage. In contrast to EC, non-embryogenic calli (NEC) consist of isodiametric, actively dividing cells. ESM of Larix sibirica contains a high content of IAA (100 times more than in NEC) and a low content of ABA. Reading of transcriptomes of EC and NEC in the culture in vitro of Larix sibirica by automated processing showed that the greatest differences in the level of expression of EC are characterized by proteins responsible for the regulation of physiological functions and associated with the development of the embryo. Thus, the markers of somatic embryogenesis of Pinaceae species are: polarization and asymmetric division of elongated cells, formation of ESM, localization of IAA in the elongated cell, high content of IAA in the ESM, as well as the presence of proteins responsible for physiological functions and embryogenesis.

Acknowledgements: The reported study was funded by Russian Foundation for Basic Research, Government of Krasnoyarsk Territory, Krasnoyarsk Region Science and Technology Support Fund to the research projects number 19-44-240009. 